\title{
The time scale for the transition to turbulence in a high Reynolds number, accelerated flow
}

\author{
H. F. Robey, Ye Zhou, and A. C. Buckingham \\ Lawrence Livermore National Laboratory, Livermore, Calfiornia 94550 \\ P. Keiter \\ Atmospheric, Oceanic, and Space Sciences, University of Michigan, Ann Arbor, Michigan 48105 \\ B. A. Remington \\ Lawrence Livermore National Laboratory, Livermore, California 94550 \\ R. P. Drake \\ Atmospheric, Oceanic, and Space Sciences, University of Michigan, Ann Arbor, Michigan 48105
}

(Received 8 August 2002; accepted 6 November 2002)

\begin{abstract}
An experiment is described in which an interface between materials of different density is subjected to an acceleration history consisting of a strong shock followed by a period of deceleration. The resulting flow at this interface, initiated by the deposition of strong laser radiation into the initially well characterized solid materials, is unstable to both the Richtmyer-Meshkov (RM) and RayleighTaylor (RT) instabilities. These experiments are of importance in their ability to access a difficult experimental regime characterized by very high energy density (high temperature and pressure) as well as large Reynolds number and Mach number. Such conditions are of interest, for example, in the study of the RM/RT induced mixing that occurs during the explosion of a core-collapse supernova. Under these experimental conditions, the flow is in the plasma state and given enough time will transition to turbulence. By analysis of the experimental data and a corresponding one-dimensional numerical simulation of the experiment, it is shown that the Reynolds number is sufficiently large $\left(\operatorname{Re}>10^{5}\right)$ to support a turbulent flow. An estimate of three key turbulence length scales (the Taylor and Kolmogorov microscales and a viscous diffusion scale), however, shows that the temporal duration of the present flow is insufficient to allow for the development of a turbulent inertial subrange. A methodology is described for estimating the time required under these conditions for the development of a fully turbulent flow. (C) 2003 American Institute of Physics.
\end{abstract}

[DOI: $10.1063 / 1.1534584]$

\section{INTRODUCTION}

An unstable flow driven by strong laser radiation is described in which the interface between two materials of dissimilar densities is first subjected to a strong shock and then decelerated over a longer time scale. The acceleration history of the interface is similar to that which occurs during the explosion of a core-collapse supernova in which the denser core materials are initially shocked and then decelerated by the surrounding lower density materials. ${ }^{1-5}$ In both the astrophysical and laboratory cases, perturbations on the interface are unstable to the Richtmyer-Meshkov (RM) ${ }^{6,7}$ and Rayleigh-Taylor (RT), ${ }^{8,9}$ instabilities. Given a large enough value of an appropriately defined Reynolds number, the growth of these interfacial instabilities will eventually cause the interface to transition to turbulence.

The Reynolds number based on the length and velocity scales of the interfacial perturbations in the present experiment is time dependent, but quickly becomes large enough $\left(\operatorname{Re}<10^{5}\right)$ to allow for the transition to turbulence. For a wide range of stationary flow geometries (shear flows, jets, wakes, boundary layers, etc.), Dimotakis ${ }^{10}$ has shown that there appears to be a nearly universal value of the Reynolds number $\left(\operatorname{Re}_{\text {mix }} \approx 2 \times 10^{4}\right)$ at which a rather abrupt transition to a well-mixed state is observed to occur. This abrupt change in flow character has been termed the "mixing transition." Since these flows exhibit relatively small changes for Reynolds numbers above this value, Dimotakis has suggested this mixing transition Reynolds number as a possible criterion for the onset of fully developed turbulence. The goal of this paper is to adapt this stationary flow criterion to the present nonstationary experiments in order to estimate the time required for the development of a fully turbulent flow.

The present experiments are designed to study the RM and RT induced mixing that occurs in the explosion phase of a core-collapse supernova. In Ryutov et al.,${ }^{11}$ the spatial and temporal scales appropriate to the supernova problem were given together with estimates of fluid properties such as the kinematic viscosity. From these, the Reynolds number was estimated to be extremely large $\left(\operatorname{Re}>10^{10}\right)$. Under these conditions, as will be discussed later, the interfacial mixing will indeed the turbulent. Ryutov also showed that a laserdriven experiment could be conducted in which a scaled interface velocity history matched that of the supernova. Since the spatial scale of the experiment is much smaller than that of the supernova, the Reynolds number is correspondingly 
smaller $\left(\operatorname{Re}=10^{5}\right)$. This value of the experiment Reynolds number is clearly large enough that the effect of viscosity is negligible on the large-scale motions involved in the interpenetration and stirring of the more-dense and less-dense fluids. As pointed out by Galmiche and Gauthier ${ }^{12}$ in a closely related flow, however, a large value of the Reynolds number is a necessary, but not sufficient condition to insure that the flow transitions to turbulence. This large value of the Reynolds number must be sustained for a long enough period of time. That a turbulent flow takes a finite period of time to develop has, of course, been well known since the pioneering work of Stokes. ${ }^{13}$ Stokes analyzed the early time solution of the Navier-Stokes equations for an impulsively accelerated, infinite flat plane boundary layer, where the balance between acceleration and viscous dissipation gives a boundary layer thickness that grows as $(\nu t)^{1 / 2}$, regardless of the value of the Reynolds number. It is only after a finite period of time, that perturbations initiated by instability mechanisms can give rise to a turbulent spectrum. In strongly accelerated flows, this time scale for the onset of turbulence can be comparable to the duration of the flow, itself.

An important consequence of an insufficient development time is seen at the smallest scales of motion, where the degree of molecular mixing may be vastly different between the full-scale problem and the scaled laboratory experiment. In the present study, we explore this temporal aspect of the problem. By adapting the mixing transition work of Dimotakis, a methodology is given for estimating the time required for the attainment of fully developed turbulence in a transitional flow at a high Reynolds number.

The outline of the paper is as follows. In Sec. II, the experimental setup and essential results are described. Section III summarizes the results of a corresponding onedimensional numerical simulation that is used to estimate the underlying fluid properties (pressure, density, temperature, degree of ionization, etc.) for the experiment. From these conditions, two important plasma transport properties, the kinematic viscosity and the binary mass diffusivity are then estimated. In Sec. IV, the experimental measurements together with the kinematic viscosity estimate are used to estimate the time-dependent value of the Reynolds number and two important turbulence length scales, the Taylor microscale and the Kolmogorov dissipation scale. The Taylor microscale is useful as an estimate of the largest scale in a turbulent spectrum at which the flow is isotropic, and therefore independent of the large-scale features driving the flow evolution. It therefore can be used as an estimate of the large-scale, low-wave-number end of a turbulent inertial range. The Kolmogorov dissipation scale provides an estimate of the small-scale, high-wave-number end of the inertial range.

These results are then used in Sec. V to develop a methodology for estimating the time required for the development of an inertial subrange in the turbulent spectrum. This is done by exploiting the well-known analogy between the Reynolds number dependence of the Taylor microscale $\left(\lambda_{T}\right.$ $\sim \operatorname{Re}^{-1 / 2}$ ) and that of a viscous laminar boundary layer ( $\delta$ $\sim \operatorname{Re}^{-1 / 2}$ ). The essential idea that is developed is that for high Reynolds number flows of short time duration, the Taylor microscale grows like $(\nu t)^{1 / 2}$ until it reaches its stationary flow value. For such time-limited flows, this viscous development scale sets the time for the appearance of an inertial subrange in the turbulent spectrum, and provides us with a means of estimating the time required for the development of a fully turbulent flow which will exhibit a mixing transition.

\section{EXPERIMENTAL SETUP AND RESULTS}

\section{A. Experimental setup}

The experiments are conducted on the Omega Laser at the Laboratory for Laser Energetics (LLE), University of Rochester. ${ }^{14-16}$ Figure 1(a) shows a three-dimensional illustration of the experimental setup that is used. The strong shock conditions of interest are achieved by directing 10 beams with a nominal measured energy of $500 \mathrm{~J} /$ beam at a laser wavelength of $0.351 \mu \mathrm{m}$ onto the target. Each beam has a super-Gaussian spatial intensity profile defined as $I / I_{0}$ $=\exp \left[-(r / 412 \mu \mathrm{m})^{4.7}\right]$. The combined spatial profile of all drive beams also follows this profile, with $I_{0}=9 \times 10^{14}$ $\mathrm{W} / \mathrm{cm}^{2}$. The intensity is reasonably constant over a central diameter of $600 \mu \mathrm{m}$ and falls off by about $10 \%$ by $800 \mu \mathrm{m}$. The target diameter, by comparison, is $800 \mu \mathrm{m}$. Since considerable laser energy extends laterally beyond the diameter of the target packages, a $75 \mu \mathrm{m}$ thick Beryllium shield with an outer diameter of $2.5 \mathrm{~mm}$ and an inner aperture of 950 $\mu \mathrm{m}$ was used to delay the propagation of a shock around the sides of the target. This proved to be successful in generating reasonably planar shock propagation through the target materials. For all experiments, the temporal pulse is nominally flat with a duration of $1 \mathrm{~ns}$.

Figure 1(b) shows a schematic of a two-dimensional slice through the center of the target. The target is mounted within a Be shock tube with an outer diameter (OD) of 1100 $\mu \mathrm{m}$ and an inner diameter (ID) of $800 \mu \mathrm{m}$. The presence of the shock tube improves the planarity of the experiment by decreasing the lateral expansion of the target materials. Beryllium was used for the shock tube material, since it is essentially transparent to the diagnostic $\mathrm{x}$ rays. The target consists of a $150 \mu \mathrm{m}$ thick polyimide layer $\left(\rho=1.41 \mathrm{~g} / \mathrm{cm}^{3}\right)$, with the remainder of the target filled with a low density carbonized resorcinol formaldyhide (CRF) foam $(\rho=0.1$ $\left.\mathrm{g} / \mathrm{cm}^{3}\right)$. Embedded within the polyimide layer is a radiographically opaque tracer strip of $4.3 \%$ brominated polystyrene $(\mathrm{CH})$ as shown at the bottom of Fig. 1(b) in an end-on view of the target. This tracer layer measures $75 \mu \mathrm{m}$ in the direction along the $\mathrm{Be}$ tube, and its $200 \mu \mathrm{m}$ wide in the transverse or diagnostic line-of-sight direction. The density of the $\mathrm{CH}(\mathrm{Br})$ layer $\left(\rho=1.42 \mathrm{~g} / \mathrm{cm}^{3}\right)$ is nearly identical to that of the surrounding polyimide. Throughout this paper, this composite layer of polyimide and brominated polystyrene will simply be referred to as the "plastic" layer. When viewed in side-illuminated radiography, most of the contrast comes from the more opaque $\mathrm{CH}(\mathrm{Br})$ tracer layer, allowing visualization of the interfacial structure over only the central $200 \mu \mathrm{m}$ of the target. This helps to eliminate wall effects that are inherent in such an integrated line-of-sight diagnostic. ${ }^{17}$ A perturbation of wavelength $\lambda=50 \mu \mathrm{m}$ and initial peak-to- 


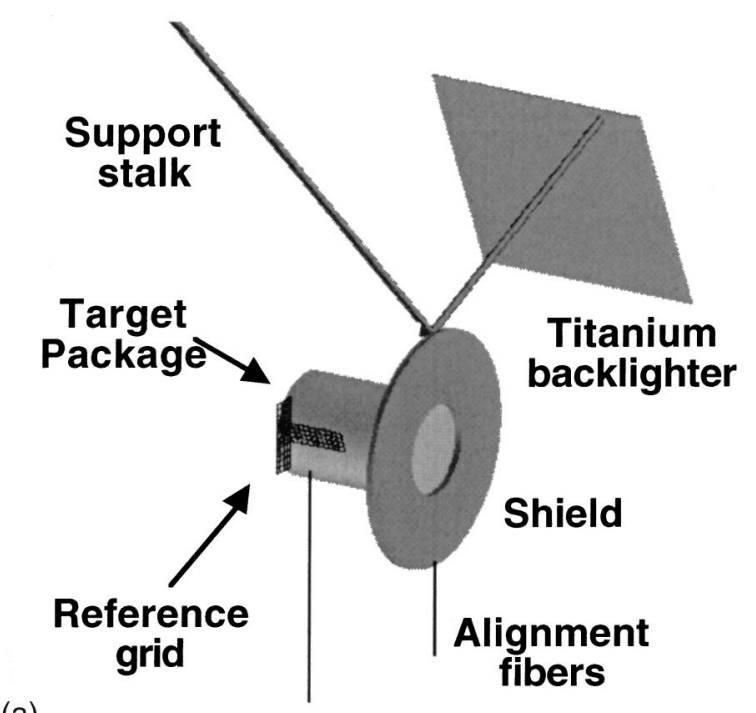

(a)

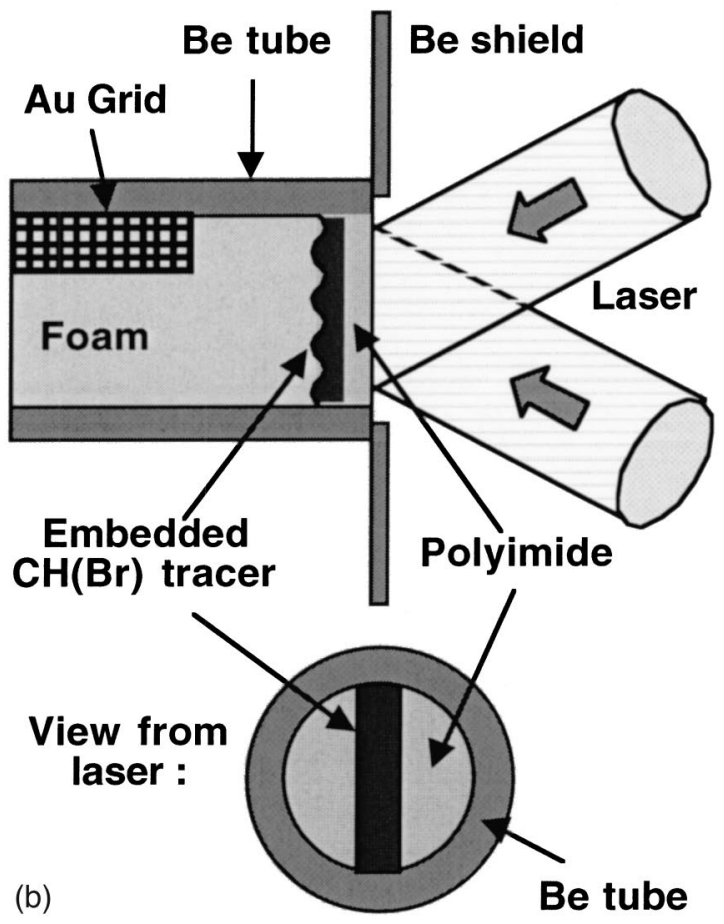

FIG. 1. (a) Three-dimensional illustration of the experimental setup. (b) Schematic of a two-dimensional slice through the center of the target.

valley amplitude $a_{\mathrm{PV}}=5 \mu \mathrm{m}$ is preimposed at the polyimidefoam interface. The evolution of the interfacial instability was diagnosed with $\mathrm{x}$ rays generated by directing an additional 7 Omega beams onto a $2.5 \mathrm{~mm}^{2}$ by $12 \mu \mathrm{m}$ thick titanium backlighter foil located $4 \mathrm{~mm}$ from the center of the target as shown in Fig. 1(a). These beams, driven by a separate oscillator, were delayed in time relative to the drive beams by up to $14 \mathrm{~ns}$ to observe the instability evolution. The contrast generated by differential absorption of the backlighter $\mathrm{x}$-rays by the target materials was imaged with a gated framing camera. ${ }^{18}$

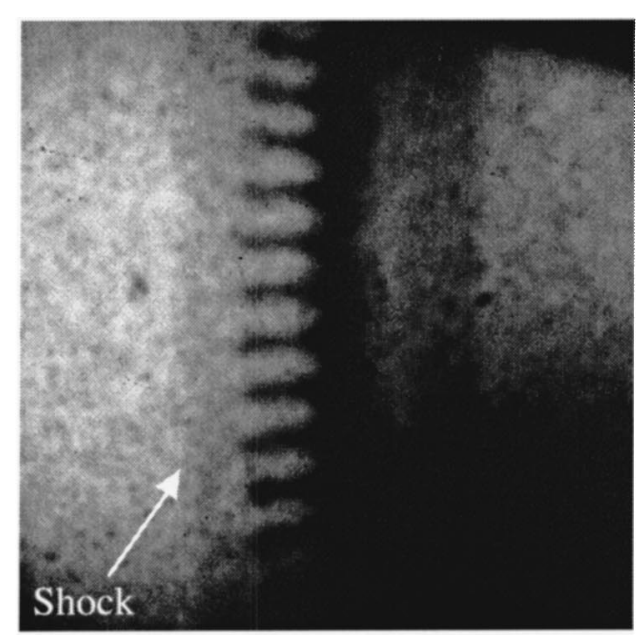

(a)

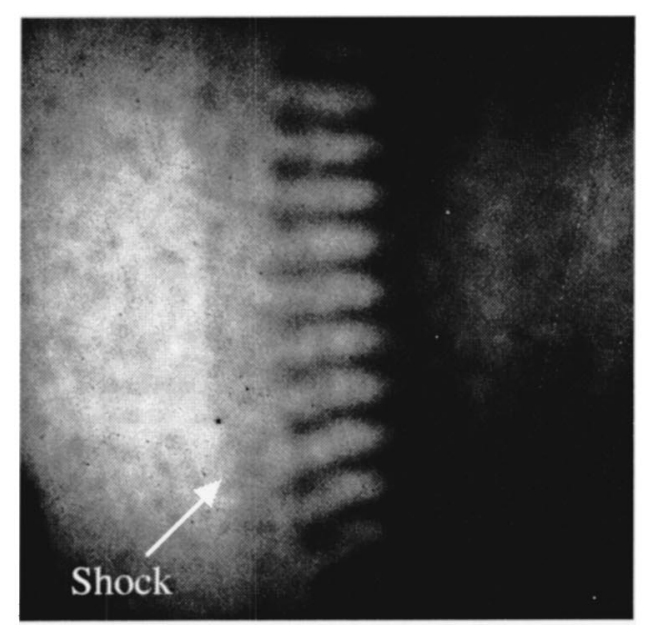

(b)

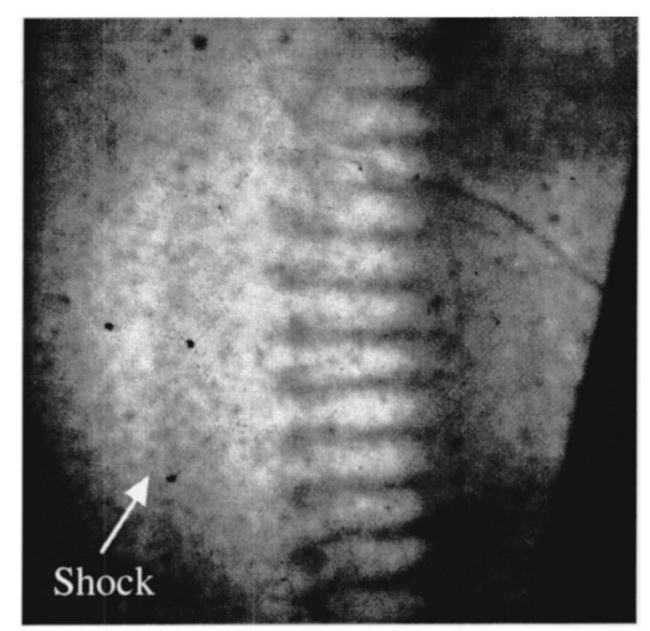

(c)

FIG. 2. Radiograph of the interface at (a) $t=8 \mathrm{ns,} \mathrm{(b)} t=12 \mathrm{ns,} \mathrm{(c)} t=14 \mathrm{~ns}$.

\section{B. Experimental results}

Experimental radiographs obtained using $4.7 \mathrm{keV} \mathrm{He}-\alpha$ $\mathrm{x}$ rays from the Ti backlighter foil are shown in Fig. 2 at $t=8$, 12 , and 14 ns. In each image, the location of the shock 
(propagating from right to left) is seen due to the difference in optical depth of the shocked and unshocked foam. The instability at the plastic-foam interface is seen as well. A reference grid consisting of $5 \mu \mathrm{m}$ Au wires with a period of $63 \mu \mathrm{m}$ was used to determine the mean interface location and the peak-to-valley amplitude of the interfacial perturbation. The perturbation amplitude clearly grows with time, but remains dominated by a single-mode structure. The appearance of a vortical roll-up at the tips of the denser polyimide spikes is seen in the later time images, though there is no clear indication that the interface has become turbulent. As will be shown, the Reynolds number based on this measured perturbation amplitude and growth velocity is sufficiently large to support a turbulent flow. It is even greater than the mixing transition Reynolds number $\mathrm{Re}_{\mathrm{mix}}$. The remainder of the paper will be aimed at providing at plausible explanation for this delayed onset of turbulence and may offer a mean of predicting the conditions required for the appearance of turbulence in this and other such strongly accelerated flows.

A one-dimensional numerical simulation of the underlying, unperturbed flow was performed using the radiation hydrodynamics code HYADES. ${ }^{19}$ Figure 3(a) shows the deceleration history of the mean interface taken from this simulation. A plot of the experimentally measured nondimensional perturbation amplitude $k a$ vs time is shown in Fig. 3 (b), where the perturbation amplitude $a$ is defined as onehalf of the full bubble-to-spike extent and is normalized by the wave number. The solid points at $t=8,12$, and $14 \mathrm{~ns}$ are taken from the images of Fig. 2, and the line is a linear fit to the experimental data.

The shock passes through the interface at $t=3 \mathrm{~ns}$. The interface inverts phase and begins to grow due to both the Richtmyer-Meshkov and Rayleigh-Taylor instabilities. The amount of RM growth is significant early in time, but quickly becomes small in comparison to the subsequent RT growth as the interface is decelerated. The relative contributions of RM and RT to the perturbation growth can be estimated by looking at the linear growth rates of the two instabilities. The initial RT and RM growth rates are $\dot{a}_{\mathrm{RT}}$ $=\sqrt{A k g} a_{0}$ and $\dot{a}_{\mathrm{RM}}=k a_{0} A u_{\text {interface }}$, respectively. In each of these, $A=\left(\rho_{\mathrm{CH}}-\rho_{\text {foam }}\right) /\left(\rho_{\mathrm{CH}}+\rho_{\text {foam }}\right)$ is the Atwood number, $g$ is the interface acceleration, and $a_{0}$ is the pre-shock perturbation amplitude. From Figs. 3(a) and 4(e), the peak interface deceleration is $10^{15} \mathrm{~cm} / \mathrm{s}^{2}$ and the Atwood number is approximately 0.55 . This gives an initial RT growth rate of $2.1 \times 10^{5} \mathrm{~cm} / \mathrm{s}$. The corresponding magnitude of the RM growth rate is $1.4 \times 10^{6} \mathrm{~cm} / \mathrm{s}$, which is 6.5 times greater than the initial RT contribution. (Note that in the estimation of the RM contribution, the pre-shock amplitude $a_{0}$ was deliberately used as an upper bound on the growth rate. In reality, the average of the pre-shock and post-shock amplitudes should be used as suggested by Meyer and Blewett. ${ }^{20}$ This will result in a smaller relative RM contribution.) Within a few nanoseconds, however, the RT contribution begins to dominate. By $3 \mathrm{~ns}$ after shock passage ( $t=6 \mathrm{~ns}$ ), for example, the $\mathrm{RM} / \mathrm{RT}$ growth rate ratio is 0.5 . Beyond this time, the experiment is dominated by Rayleigh-Taylor growth, and the RM contribution is small.
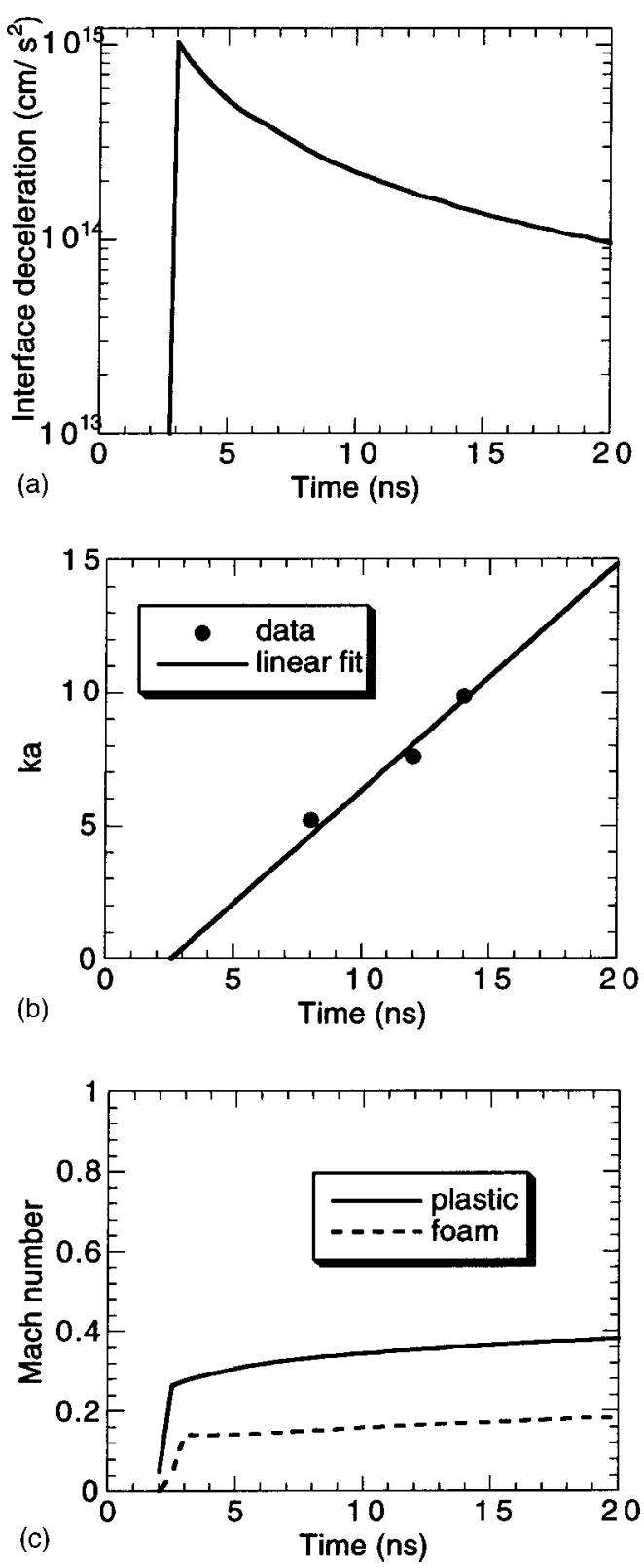

FIG. 3. (a) Time history of the mean interface deceleration. (b) Nondimensional perturbation amplitude vs time. (c) Perturbation Mach number vs time.

At $t=3 \mathrm{~ns}$, the interface begins to decelerate, and exponential RT growth initially occurs. When the perturbation amplitude becomes comparable to the wavelength $(k a \sim 1)$, the perturbation growth rate saturates to a constant value. ${ }^{21}$ As Fig. 3(b) shows, the exponential growth phase ends very quickly, within $1 \mathrm{~ns}$ of the shock arrival time. The remainder of the perturbation growth occurs in the saturated nonlinear phase and is characterized by a constant growth rate. A linear fit to the data of Fig. 3(b) gives a saturated RT growth rate $d a / d t$ of approximately $6.8 \mu \mathrm{m} / \mathrm{ns}$. As this is a strongly shocked flow, it is of interest to assess the effect of compressibility on the perturbation growth. The role of compressibility is characterized by a Mach number formed from the ratio of the perturbation growth velocity and the local sound speed as determined from the HYADES simulation, 

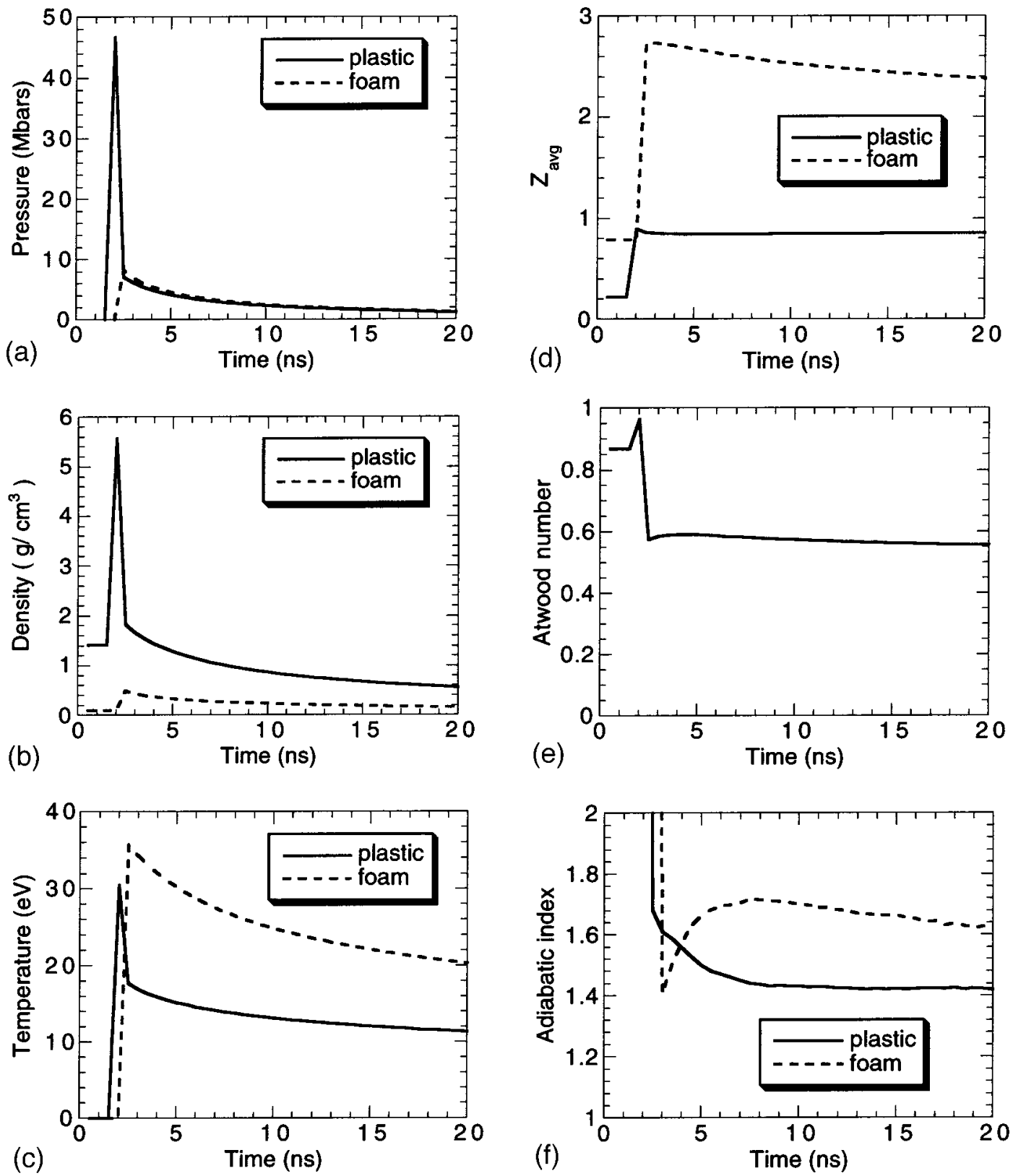

FIG. 4. Numerical simulation of the temporal history of (a) pressure, (b) density, (c) temperature, (d) degree of ionization, (e) Atwood number, and (f) adiabatic index.

$M=(d a / d t) / \sqrt{\gamma P / \rho}$. The flow parameters $P, \rho$, and $\gamma$ are given in the next section for both the more-dense plastic and less-dense foam side of the interface. The resulting perturbation Mach number is plotted in Fig. 3(c). It differs on either side of the interface due to the difference in sound speeds of the two materials. After passage of the shock this perturbation Mach number increases rather slowly, and remains below 0.4 throughout the duration of the experiment. The effects of compressibility on the perturbation development are therefore expected to be rather small.

\section{ESTIMATION OF TRANSPORT PARAMETERS}

\section{A. Plasma kinematic viscosity}

In order to determine the Reynolds number or estimate any of the length scales of importance to the description of a turbulent flow, we must first determine an appropriate value for the kinematic viscosity. The simplest formula for the estimation of the viscosity of a plasma is that given by Braginskii $^{22}$ which is applicable to the low density, high temperature plasma state where ionic coupling is weak. The coupling is determined by the plasma parameter $\Gamma$ $=Z^{2} e^{2} /\left(k_{B} T \lambda_{i}\right)$, where $Z e$ is the ionic charge and $\lambda_{i}$ $=\left(3 / 4 \pi N_{i}\right)^{1 / 3}$ is the average interionic distance $\left(N_{i}\right.$ is the ion number density in $\left.\mathrm{cm}^{-3}\right)$. $\Gamma$ is therefore a measure of the ratio of potential to kinetic energy of the plasma. For $\Gamma \leqslant 1$, the plasma ions are weakly coupled, and the formula of Braginskii for the kinematic viscosity is given as

$$
\nu_{i}=3.3 \times 10^{-5} \frac{\sqrt{A} T^{5 / 2}}{\ln (\Lambda) Z^{4} \rho_{i}} .
$$


Here, $\nu$ is the kinematic viscosity in $\mathrm{cm}^{2} / \mathrm{s}, T$ is the ion temperature measured in $\mathrm{eV}, \rho$ is the density in $\mathrm{g} / \mathrm{cm}^{3}, A$ and $Z$ are the atomic weight and number, and $\ln (\Lambda)$ is the Coulomb logarithm, which is again a function of temperature and degree of ionization. For numerical evaluation of the kinematic viscosity, we use the Coulomb logarithm definition given in the NRL Plasma Formulary. ${ }^{23}$

For dense plasma conditions or for conditions where mixing between two ionic species is involved, we also use the more extensive viscosity model of Clerouin et al. ${ }^{24}$ which is applicable over a wide range of temperatures and densities than the Braginskii model. For this model, the kinematic viscosity is given by

$$
\begin{aligned}
\nu\left(\mathrm{cm}^{2} / \mathrm{s}\right)= & 6.55 \times 10^{-10} Z_{\mathrm{eff}} m_{i}^{1 / 2} n_{i}^{5 / 6} \\
& \times\left\{\begin{array}{cc}
1.1 \Gamma_{\mathrm{eff}}^{-1.895}, & \Gamma_{\mathrm{eff}}<2, \\
\lambda I_{1}+\frac{\left(1+\lambda I_{2}\right)}{\lambda I_{3}}, & 2<\Gamma_{\mathrm{eff}}<160,
\end{array}\right.
\end{aligned}
$$

where the parameter $\lambda$ and fits to the integrals $I$ are given by

$$
\begin{aligned}
& \lambda=\frac{4 \pi}{3}\left(3 \Gamma_{\text {eff }}\right)^{3 / 2}, \\
& I_{1}=\left(180 \pi^{3 / 2} \Gamma_{\text {eff }}\right)^{-1}, \\
& I_{2}=\left(1 / 60 \pi^{2}\right)\left(0.49-2.23 \Gamma_{\text {eff }}^{-1 / 3}\right), \\
& I_{3}=\left(1 / 10 \pi^{3 / 2}\right)\left(2.41 \Gamma_{\text {eff }}^{1 / 9}\right) .
\end{aligned}
$$

Here $Z_{\text {eff }}=x_{1} Z_{1}+x_{2} Z_{2}$ is the number-density-weighted average charge for a binary ionic mixture of two species with number densities $x_{1}$ and $x_{2}$. The effective plasma coupling parameter for this mixture is

$$
\Gamma_{\mathrm{eff}}=\frac{e^{2} Z_{\mathrm{eff}}^{1 / 3} \overline{Z^{5 / 3}}}{r_{i} k_{B} T}, \quad \overline{Z^{5 / 3}}=x_{1} Z_{1}^{5 / 3}+x_{2} Z_{2}^{5 / 3},
$$

where $r_{i}$ is the mean ionic radius, and $k_{B}$ is the Boltzmann constant. This model uses an extended form of the Wallenborn-Baus formula, ${ }^{25}$ which was originally developed for a one-component plasma. Clerouin et al. have extended this model in two regards. First, they have provided a least-squares fit to the weak coupling calculations of Wallenborn and Baus to extend the formula to the regime $\Gamma<2$ [top part of Eq. (2)]. More importantly, they have performed molecular dynamics simulations of plasma mixtures of dissimilar species to demonstrate that the effective coupling parameter $\Gamma_{\text {eff }}$ is indeed the appropriate parameter to use in the calculation of the transport properties of mixtures. The resulting model gives results in good agreement with the model of Braginskii in the low-density regime where both are applicable.

We can now evaluate the flow conditions for the laser experiment discussed in the previous section. Figure 4 shows the time history of the basic fluid parameters of interest. These are again taken from the one-dimensional HYADES simulation. Figures 4(a)-4(d) show the pressure, density, temperature, and degree of ionization, respectively, for one computational zone on either side of the density discontinuity. For all plots in Fig. 4, the solid line is the density in the
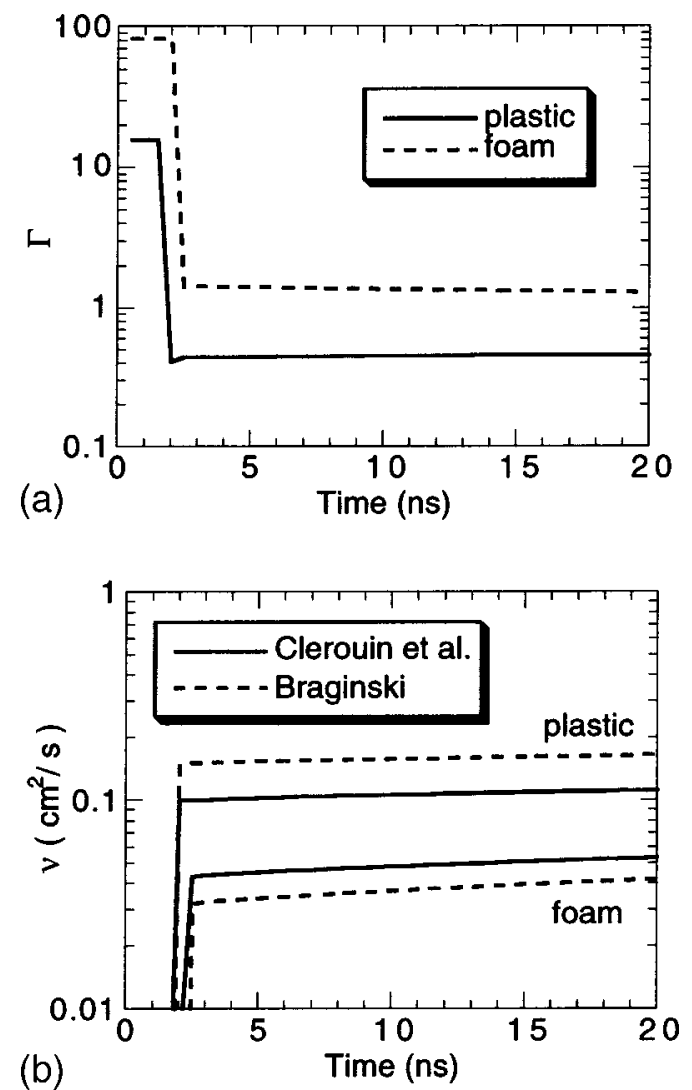

FIG. 5. Numerical simulation of the temporal history of (a) the plasma coupling parameter $\Gamma$, and (b) the kinematic viscosity.

plastic layer (polyimide or brominated polystyrene) and the dashed line gives the density in the carbon foam. Figures 4(e) and 4(f) show plots of two important related parameters. Figure 4(e) gives the Atwood number $A$, which after shock passage remains approximately constant at $0.55-0.6$. The Atwood number is a critical parameter for both the RM and RT instabilities. Figure 4(f) shows a plot of the adiabatic index, $\gamma$, which was previously used to estimate the Mach number at the interface. In Fig. 4(f), the adiabatic index is estimated from the pressure and density as $\gamma=d(\ln p) / d(\ln \rho)$. It is again plotted for both sides of the interface. After passage of the shock, $\gamma$ settles down for both materials to a value between 1.4-1.6.

From the basic parameters of Figs. 4(a)-4(d), the plasma coupling parameter $\Gamma$ can be calculated. This is plotted in Fig. 5(a). After passage of the shock, the plasma parameter is seen to be in the "uncomfortable" range close to one, i.e., neither in the weakly coupling regime where kinetic theory is valid $(\Gamma \leqslant 1)$ nor the very strongly coupled regime $(\Gamma \gg 1)$ where molecular dynamics simulations can provide rigorous transport properties. ${ }^{26}$

The kinematic viscosity, calculated using both the Braginskii formula (dashed curves) as well as that of Cherouin et al. (solid curves), is plotted in Fig. 5(b). Again the viscosity is plotted for both sides of the interface. After passage of the shock, $\nu$ remains reasonably constant at approximately $0.1-0.2 \mathrm{~cm}^{2} / \mathrm{s}$ for the denser plastic layer and $0.04-0.05$ $\mathrm{cm}^{2} / \mathrm{s}$ for the carbon foam. It is interesting to observe that the lower density foam has a lower kinematic viscosity than the 
plastic. The reason for this can be understood from the simple analytic form of the Braginskii formula of Eq. (1). Even though the carbon foam has a higher temperature [Fig. 4(c)] and lower density [Fig. 4(b)] than the plastic (both of which would result in a higher kinematic viscosity), the strong dependence on the degree of ionization $\left(Z^{4}\right)$ results in a lower value of the kinematic viscosity for the foam. The Clerouin model gives the same comparative result, though it is not obvious from the more complicated analytic form of this model.

\section{B. Plasma binary mass diffusivity}

An additional transport term that can strongly affect the flow development is the binary mass diffusivity. As was shown by Duff et al. ${ }^{27}$ mass diffusivity affects the growth rate dispersion relation of perturbations on a RayleighTaylor unstable interface, eventually causing stability at sufficiently large wave number. For the range of plasma coupling conditions of interest $(\Gamma \sim 1)$, the method of Paquette et $a l .{ }^{26}$ can be used. In this method, the diffusion coefficients are obtained as high-accuracy analytic fits to numerically evaluated collision integrals for a screened Coulomb potential. By contrast with the method of Clerouin used for estimating plasma viscosity, the method of Paquette is rigorously valid in the dilute plasma regime and is extrapolated to the dense plasma regime. Again, by comparison with molecular dynamics simulations in the dense plasma regime, the method is shown to provide reasonable estimates in the intermediate region $(\Gamma \sim 1)$ of interest to the present experiments.

Figure 6(a) shows a plot of the binary mass diffusivity $D_{12}$ at the interface as a function of time. After passage of the shock, $D_{12}$ remains reasonably constant at approximately $0.08 \mathrm{~cm}^{2} / \mathrm{s}$. The numerical values are observed to be quite close to those of the kinematic viscosity, falling right between the values of $\nu$ on either side of the interface. The Schmidt number, $\mathrm{Sc}=v_{\mathrm{avg}} / D_{12}$, quantifying this comparison between diffusion of momentum and mass is plotted in Fig. 6(b). For this plot, the average of the kinematic viscosities (taken for the Clerouin model) on either side of the interface is used. Prior to shock arrival, the Schmidt number is rather large $(\mathrm{Sc}=32)$. There is probably considerable uncertainty in this value as the temperature from the HYADES simulation before shock arrival is of order $0.05 \mathrm{eV}$, which is very comparable to the plastic melting temperature. After the shock, however, Sc is observed to be of order one, which is characteristic of a wide range of gasses and plasmas. As Fig. 6(b) shows, this experiment, while being complicated somewhat by a nonconstant acceleration and the effects of decompression, does have the nice feature of providing reasonably constant transport properties characterized by $\nu, D_{12}$, and Sc.

\section{TURBULENT LENGTH SCALES}

Having determined the appropriate dissipative transport properties, it is now interesting to look at some of the length scales of importance in studying the onset of turbulence in the experiment. We begin with an estimate of the Reynolds number. Using the measured peak-to-valley amplitude of the

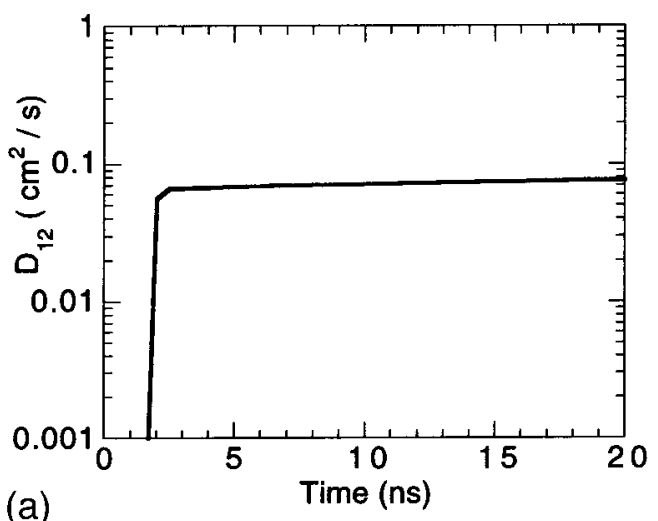

(a)

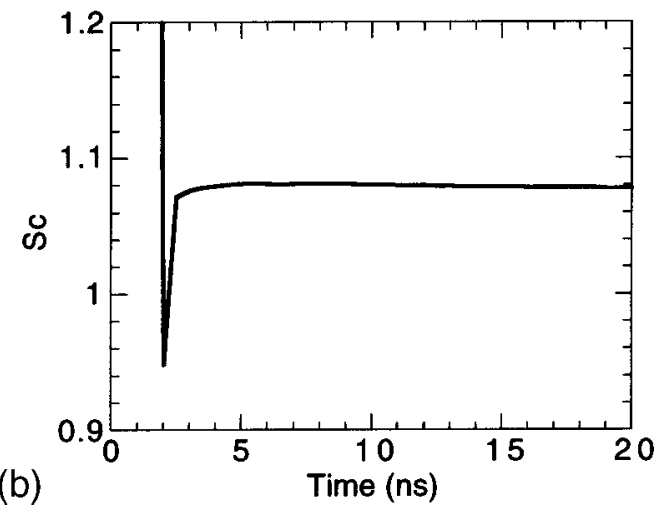

FIG. 6. (a) Binary mass diffusivity and (b) Schmidt number at the interface vs time.

perturbation $L$ from Fig. 3(b) and the constant RT growth velocity difference across this layer as the characteristic length and velocity scales together with the viscosity (Clerouin model) given in Fig. 5(b), a Reynolds number $\operatorname{Re}_{L}$ can be formed. The Reynolds number is evaluated using kinematic viscosity values on either side of the interface. This is plotted in Fig. 7. The Reynolds number is increasing with time, as this is a temporally developing flow. The final Reynolds number is seen to be well above the mixing transition Reynolds number of $2 \times 10^{4}$, which forms an effective crite-

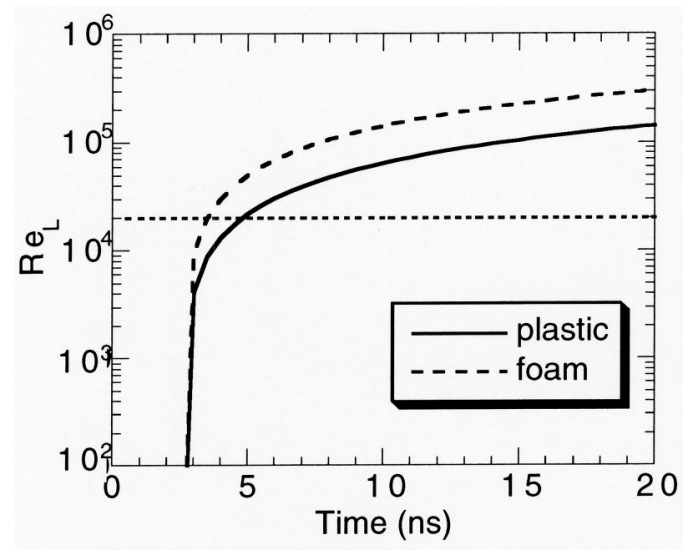

FIG. 7. Temporal evolution of the Reynolds number based on the measured amplitude and growth rate of the interfacial perturbation and the calculated value of the kinematic viscosity. The dashed line is the mixing transition Reynolds number of Dimotakis. 
rion for fully developed turbulence in a wide range of stationary flow geometries. The important distinction here is that the present flow is not stationary, but transitional.

Even though the flow is transitional, we can still estimate some important length scales such as the Taylor microscale $\lambda_{T}{ }^{28}$ and the Kolmogorov dissipation scale $\lambda_{k}$. These scales are estimated based on the integral scale Reynolds number as

$$
\frac{\lambda_{k}}{L}=\operatorname{Re}_{L}^{-3 / 4} \text { and } \frac{\lambda_{T}}{L}=\operatorname{Re}_{L}^{-1 / 2} .
$$

These are to be thought of as the dissipation scale and Taylor microscale that would result at this Reynolds number if sufficient time were to be made available for development of this flow to a stationary state.

\section{DISCUSSION}

In order to provide an estimate of the time required for the onset of turbulence, we propose the following modification to the idea put forth by Dimotakis. Dimotakis showed that the appearance of a mixing transition to a welldeveloped turbulent state was consistent with the appearance of a range of scales that were decoupled from both the largescale driving effects as well as the small-scale viscous effects. Specifically, this range of scales was identified as

$$
\frac{\lambda_{\nu}}{L}=50 \operatorname{Re}_{L}^{-3 / 4}<\frac{\lambda}{L}<\frac{\lambda_{\mathrm{LT}}}{L}=5.0 \mathrm{Re}_{L}^{-1 / 2},
$$

where $\lambda_{\nu}=50 \lambda_{K}$ is an inner viscous scale well above the Kolmogorov dissipation scale $\lambda_{K}$, and $\lambda_{\mathrm{LT}}$, which Dimotakis calls the Liepmann-Taylor scale, is characteristic of the internal laminar layers generated by viscous diffusion along the boundaries of a large-scale feature of size $L$. These two scales effectively form the lower and upper wavelength limits of the inertial subrange. In the present flow, the Liepmann-Taylor scale would correspond to the development of laminar viscous diffusional layers growing along the boundaries of the spikes of the more dense material interpenetrating the lower density carbon foam. The essential modification suggested by the present transitional flow is that the Liepmann-Taylor scale is now time dependent. The temporal development of such a laminar viscous layer is well known from the early work of Stokes ${ }^{13}$ and Blasius ${ }^{29}$ to go as $(\nu t)^{1 / 2}$ (see also Ref. 30).

We therefore propose an extension to the mixing transition work of Dimotakis by determining the experiment time at which the developing viscous diffusion scale is large enough and the Kolmogorov dissipation scale is small enough that a range of scales will exist in between the two. Since this range of scales is by definition decoupled from both the large-scale forcing effects as well as the small-scale dissipative effects, it is essentially the turbulent inertial subrange.

Figure 8 compares the temporal development of these scales for the conditions of the present experiment. The bold solid line is the Liepmann-Taylor scale $\lambda_{\mathrm{LT}}$, the thin solid line is the inner scale $\lambda_{\nu}=50 \lambda_{K}$, and the dashed line gives the developing viscous diffusion scale $5(\nu t)^{1 / 2}$. The value of the numerical constant depends somewhat on the particular

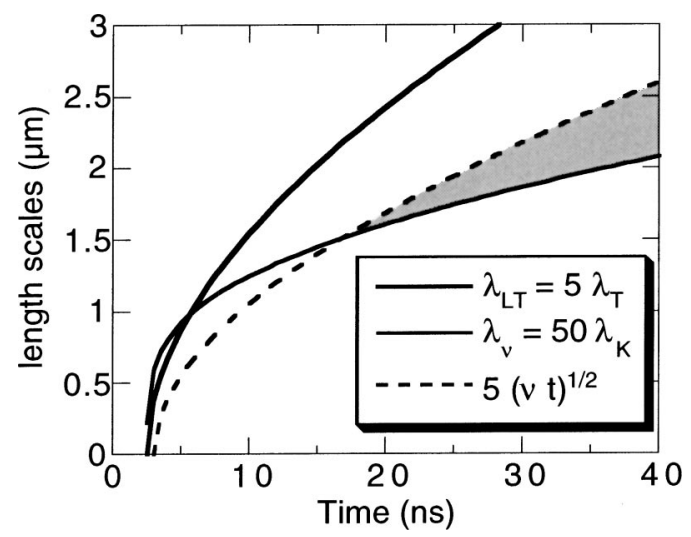

FIG. 8. A comparison of length scales relevant to the onset of turbulence. The bold line is the Liepmann-Taylor scales, the thin solid line is 50 times the Kolmogorov scale, and the dashed line is the viscous diffusion length scale. The intersection of the two solid lines occurs at the stationary flow mixing transition Reynolds number of Dimotakis. The shaded region indicates the region where an inertial subrange can exist in this developing flow.

flow studied. Initially after the passage of the shock, the Reynolds number is small and the inner scale $\lambda_{\nu}$ exceeds both the Liepmann-Taylor scale and the viscous diffusion scale. At $t \approx 6 \mathrm{~ns}$, the curves of $\lambda_{\mathrm{LT}}$ and $\lambda_{\nu}$ intersect. This occurs at a Reynolds number of order $10^{4}$, roughly the Dimotakis mixing transition value. For a stationary flow, this would indicate a sufficient separation of scales to allow for a mixing transition to occur. In our experiment, however, there has not been sufficient time to allow for the full development of the Liepmann-Taylor scale, and the appropriate scale to use is the viscous diffusion scale, which is somewhat smaller. At $t=17 \mathrm{~ns}$, the dashed curve of the viscous diffusion scale crosses the inner scale in Fig. 8. It is only at this time that a sufficient separation of scales (shaded region) will occur for this developing flow, and an inertial range will exist. This is the critical time required for the onset of a mixing transition in this flow.

It is of interest to note that the value of the Reynolds number in this experiment required for the occurrence of a mixing transition is about a factor of 10 greater than that required for a stationary flow. This is due to the finite time scale for development of the turbulent spectrum, which in this experiment is greater than the duration of the experimental observations.

A few comments are in order concerning the role of the initial spectrum of the perturbation. While we have focused on one specific experiment, the present method is general in that it depends only on two things: (1) the temporal history of the integral scale and (2) the kinematic viscosity. The initial spectrum enters through its effect on the growth of the integral scale. The integral scale development will differ for single mode experiments with different initial wavelengths, for example. It will differ for single mode vs multimode experiments, etc. The present method does not specify the time history of the integral scale (a quantity which can be determined by numerical simulation, analytic theory, or experimental measurement). Rather it uses a predetermined history to predict the time of onset of the mixing transition (a quantity which cannot be readily computed or predicted ana- 
lytically). In the present flow, the time history of the integral scale is obtained from experimental observation.

Returning to the original motivation for this work, this same procedure can now be applied to the conditions of the full-scale supernova. From Ryutov, ${ }^{11}$ parameters were given for a time of 2000 seconds after core bounce. The integral length scale is $9 \times 10^{10} \mathrm{~cm}$, the Reynolds number is 2.6 $\times 10^{10}$, and the kinematic viscosity is $7 \times 10^{7} \mathrm{~cm}^{2} / \mathrm{s}$. From these values, the time that is required for the evolution of a fully developed turbulent spectrum is found by setting $\lambda_{\nu}$ $=5(\nu t)^{1 / 2}$ and solving for $t$. The required time is found to be a mere 2.8 seconds. The full-scale problem can therefore confidently be characterized as fully turbulent, whereas the scaled experiment is only marginal in this regard.

This methodology suggests that the experiments described in this paper are perhaps very close though somewhat short of the threshold value required for the onset of a mixing transition. One must be cautious with such predictions, however, since the above analysis has applied the results of many years of stationary flow experiment and analysis to an explicitly nonstationary, transitional flow. Future experiments that follow the interface evolution to longer times should be able to achieve the required spectral development, and will be essential in verifying these results. Certainly, with the availability of megajoule-class lasers such as the National Ignition Facility, the development of accelerated flows driven by strong shocks can be studied well into the fully turbulent regime.

\section{ACKNOWLEDGMENTS}

This work was performed under the auspices of the U.S. Department of Energy by the University of California, Lawrence Livermore National Laboratory under Contract No. W-7405-Eng-48.

${ }^{1}$ J. Kane, D. Arnett, B. A. Remington, S. G. Glendinning, J. Castor, R. Wallace, A. Rubenchik, and B. A. Fryxell, Astrophys. J. Lett. 478, L75 (1997).
${ }^{2}$ B. A. Remington, J. Kane, R. P. Drake et al., Phys. Plasmas 4, 1994 (1997).

${ }^{3}$ J. Kane, D. Arnett, B. A. Remington, S. G. Glendinning, G. Bazan, R. P. Drake, B. A. Fryxell, R. Teyssier, and K. Moore, Phys. Plasmas 6, 2065 (1999).

${ }^{4}$ J. Kane, D. Arnett, B. A. Remington, S. G. Glendinning, G. Bazan, E. Müller, B. A. Fryxell, and R. Teyssier, Astrophys. J. 528, 989 (2000).

${ }^{5}$ H. F. Robey, J. O. Kane, B. A. Remington, R. P. Drake, O. A. Hurricane, H. Louis, R. J. Wallace, J. Knauer, P. Keiter, D. Arnett, and D. D. Ryutov, Phys. Plasmas 8, 2446 (2001).

${ }^{6}$ R. D. Richtmyer, Commun. Pure Appl. Math. 13, 297 (1960).

${ }^{7}$ E. E. Meshkov, Izv. Acad. Sci. USSR Fluid Dyn. 4, 101 (1969).

${ }^{8}$ Lord Rayleigh, Scientific Papers II (Cambridge University Press, England, 1900), p. 200.

${ }^{9}$ G. I. Taylor, Proc. R. Soc. London, Ser. A 201, 192 (1950).

${ }^{10}$ P. E. Dimotakis, J. Fluid Mech. 409, 69 (2000).

${ }^{11}$ D. D. Ryutov, R. P. Drake, J. Kane, E. Liang, B. A. Remington, and M. W. Wood-Vasey, Astrophys. J. 518, 821 (1999).

${ }^{12}$ D. Galmiche and S. Gauthier, Jpn. J. Appl. Phys. 35, 4516 (1996).

${ }^{13}$ G. G. Stokes, Cambr. Phil. Trans. IX, 8 (1851).

${ }^{14}$ J. M. Soures, R. L. McCrory, C. P. Verdon et al., Phys. Plasmas 3, 2108 (1996).

${ }^{15}$ T. R. Boehly, D. L. Brown, R. S. Craxton et al., Opt. Commun. 133, 495 (1997).

${ }^{16}$ D. K. Bradley, J. A. Delettrez, R. Epstein et al., Phys. Plasmas 5, 1870 (1998).

${ }^{17}$ M. Brouillette and R. Bonazza, Phys. Fluids 11, 1127 (1999).

${ }^{18}$ K. S. Budil, T. S. Perry, P. M. Bell, J. D. Hares, P. L. Miller, T. A. Peyser, R. Wallace, H. Louis, and D. E. Smith, Rev. Sci. Instrum. 67, 485 (1996).

${ }^{19}$ J. T. Larsen and S. M. Lane, J. Quant. Spectrosc. Radiat. Transf. 51, 179 (1994).

${ }^{20}$ K. A. Meyer and P. J. Blewett, Phys. Fluids 15, 753 (1972).

${ }^{21}$ S. Haan, Phys. Rev. A 39, 5812 (1989).

${ }^{22}$ S. I. Braginskii, in Reviews of Plasma Physics (Consultants Bureau, New York, 1965), Vol. 1, p. 205.

${ }^{23}$ J. D. Huba, NRL Plasma Formulary (Naval Research Laboratory, Washington, DC, 1998).

${ }^{24}$ J. G. Clerouin, M. H. Cherfi, and G. Zerah, Europhys. Lett. 42, 37 (1998).

${ }^{25}$ J. Wallenborn and M. Baus, Phys. Rev. A 18, 1737 (1978).

${ }^{26}$ C. Paquette, C. Pelletier, G. Fontaine, and G. Michaud, Astrophys. J., Suppl. Ser. 61, 177 (1986).

${ }^{27}$ R. E. Duff, F. H. Harlow, and C. W. Hirt, Phys. Fluids 5, 417 (1962).

${ }^{28}$ G. I. Taylor, Proc. R. Soc. London, Ser. A 151, 421 (1935).

${ }^{29}$ H. Blasius, Z. Math. Phys. 56, 1 (1908).

${ }^{30} \mathrm{H}$. Tennekes and J. L. Lumley, A First Course in Turbulence (MIT Press, Cambridge, MA, 1994), p. 65 